\title{
Buffy Coat Immunotranscriptomics for Diagnosis of Malignant Pleural Mesothelioma
}

\author{
Gerardo Velez*, Harvey Pass, Michele Carbone, Haining Yang, Chandra Goparaju
}

Department of Cardiothoracic Surgery, NYU Langone Health, New York, USA

Email address:

gerardo.velez@nyumc.org (G. Velez), harvey.pass@nyumc.org (H. Pass)

${ }^{*}$ Corresponding author

\section{To cite this article:}

Gerardo Velez, Harvey Pass, Michele Carbone, Haining Yang, Chandra Goparaju. Buffy Coat Immunotranscriptomics for Diagnosis of Malignant Pleural Mesothelioma. Cancer Research Journal. Vol. 9, No. 1, 2021, pp. 61-70. doi: 10.11648/j.crj.20210901.18

Received: February 16, 2021; Accepted: March 5, 2021; Published: March 12, 2021

\begin{abstract}
Malignant pleural mesothelioma is a highly aggressive tumor primarily caused by asbestos exposure and associated with poor clinical outcomes. The availability of a robust non-invasive test for the screening of asbestos-exposed subjects is therefore an important unmet clinical need. It is widely recognized that the immune system can play a fundamental role in the control of tumor growth within an organism. Simultaneously, the presence of cancer cells can activate different immune cells to undergo various phenotypic and functional changes. Buffy coat - a novel circulating microenvironment of leukocytes and platelets - may thus reflect critical immuno-oncologic activity, pathways, and molecular programs. We hypothesized the immunotranscriptome of circulating buffy coat could accurately distinguish malignant pleural mesothelioma from high-risk asbestos exposure. Buffy coat RNA was extracted from 84 patients having resection: 40 patients with malignant pleural mesothelioma and 44 patients with asbestos exposure. Gene expression profiling was performed using a Pan Cancer Immune Panel for 770 immune genes and cytokines, and significantly different genes between cohorts were selected to develop diagnostic models. Using 1000 loops of cross validation, a 37 gene signature separated malignant pleural mesothelioma from asbestos exposure with a mean validation AUCS of 0.925. Our buffy coat immunotranscriptomic signature is at least comparable to the most commonly used blood-based diagnostic biomarker for MPM, serum mesothelin-related peptide. In addition, immunophenotyping and pathway analysis of differentially expressed genes characterized MPM buffy coat as a relatively tumorigenic and immunosuppressive state. Several of the most differentially expressed genes encode proteins implicated in cancer development (e.g., CD63, CD44, ISG15, CD59, IL1R2, and TAPBP) and may hold clinical value as therapeutic targets. Larger studies on externally validated cohorts are needed to refine the signature for clinical relevance and develop a more robust diagnostic panel.
\end{abstract}

Keywords: Mesothelioma, Asbestos, Buffy Coat, Transcriptomics, Gene Expression, Biomarker, Liquid Biopsy

\section{Introduction}

Malignant pleural mesothelioma (MPM) is an aggressive neoplasm originating from the mesothelial cells lining the pleura and is causally associated with previous asbestos exposure [1]. Malignant pleural mesothelioma has a median latency time of 40-50 years between first exposure to asbestos and its diagnosis. The overwhelming majority of patients present with advanced disease and die within 2 years [2]. Current imaging and biopsy standards for detecting MPM are costly, invasive, and/or lack specificity [3, 4]. The availability of a robust non-invasive test for the screening of asbestos-exposed subjects is therefore an important unmet clinical need.
In an appropriate clinical setting, tumor biomarkers can play a meaningful role in diagnosis and screening for the early detection of cancer. Biomarkers can also provide a detailed picture of the natural history of the disease, a more comprehensive sense of tumor burden, clues about clonal evolution, or susceptibility to treatments [5]. Over the past few decades, an ever-increasing list of new biomarkers, including messenger RNA, DNA, microRNA, and antibody targets, have been investigated for mesothelioma.

It is widely recognized that the immune system can play a fundamental role in the control of tumor growth within an organism [6]. Simultaneously, the presence of cancer cells can activate different immune cells to undergo various phenotypic and functional changes. Blood acts as a dynamic solvent of 
immune activity and may thus reflect critical immuno-oncologic activity, pathways, and molecular programs [7]. Numerous biomarker studies have attempted to detect the presence of cancer by profiling gene expression in either whole blood [8-10] or peripheral blood mononuclear cells (PBMCs) [11-13]. PBMCs largely consist of lymphocytes and macrophages, and while they play a significant role in the immune system, they remain a subset of all circulating immune cells and do not include other cell types such as eosinophils and neutrophils. Considering the number of immune related pathways influenced by granulocytes $[14,15]$ and platelets $[16$, 17], we elected to explore a more global representation of the circulating microenvironment by studying the buffy coat (BC). Buffy coat is a heterogeneous mix of all circulating leukocytes, as well as platelets. Buffy coat is easily accessible but relatively unexplored regarding surrogate diagnosis of mesothelioma. Using the NanoString digital PCR platform we hypothesized that the immunotranscriptome of circulating buffy coat could accurately distinguish MPM from high-risk asbestos exposure (AEx).

\section{Methods}

\subsection{Patient Cohorts and Buffy Coat Archiving}

Forty patients with histologically proven MPM and 44 AEx members of the New York Pipe Fitters Union were consented to an NYU Langone Health IRB approved Protocol (8896) for archiving of plasma, buffy coats, and peripheral blood mononuclear cells. BC were processed within 4 hours using the NCI/NIH Early Detection Research Network Standard Operating Procedures and were frozen in sterile $250 \mathrm{ul}$ nunc vials and stored at $-80^{\circ} \mathrm{C}$.

\subsection{RNA Extraction}

BC samples were extracted using Quick-RNA Kits (ZYMO Research). RNA quantity and quality were assessed by Nanodrop OneC (Thermo Fisher Scientific). A total of $300 \mathrm{ng}$ of RNA was used for Nanostring assay.

\subsubsection{NanoString Assay}

NanoString nCounter is a multiplex nucleic acid hybridization technology that enables reliable and reproducible assessment of the expression of up to 800 genes or 228 gene fusions in 12 samples in a single assay. The nCounter platform consists of a Prep Station and Digital Analyzer. The technology is based on fluorescent molecular reporter barcodes and biotin-labeled capture probes representing the target genes for the assay. The nCounter XT CodeSet Gene Expression assay (NanoString Technologies, Seattle, WA) PanCancer Immune Profiling Panel V1.1 was selected for the quantitative assessment of expression of 730 immune genes from the buffy coats [18]. In addition to 730 genes, there were 40 housekeeping genes.

\subsubsection{Quality Control and Normalization of Gene Expression Values}

The generated raw data as RCC files were quality controlled through the NanoString nSolver QC process. The gene expression counts were normalized to the internal housekeeping genes after the background subtraction to correct any differences in the nucleic acid quality and input, and the physiological conditions across the runs. Samples that had large normalization factors or low normalization quality were excluded. Raw counts below 25 were considered background level, while those below 100 are semi-quantitative, and samples with a large portion of genes below 100 counts need to be excluded from analysis. Housekeeping genes were selected by the geNorm algorithm [20]. The geometric mean of the Housekeeping genes which exhibited a low percent coefficient of variation $(\leq 70 \%)$ was used as a normalization factor for each sample.

\subsubsection{Cell Type Immunophenotyping}

Cell type profiling scores were generated for immune cell types using expression levels of cell-type specific mRNAs as described in the literature [19]. The genes used for immune cell scoring comprise a subset of high confidence markers validated by co-expression patterns via a large survey of samples from The Cancer Genome Atlas ( $\mathrm{N}=9986)$, and confirmed by nCounter and protein analysis. The cell type score itself is calculated as the mean of the $\log 2$ expression levels for all the probes included in the final calculation for that specific cell type. The scores for the cohorts are dependent on probe specific counting and capturing efficiencies and therefore were interpreted as relative cell abundance values compared to the same cell type within the other cohort, i.e AEx vs MPM. The scores are not used as measures for the abundance of a cell type relative to other cell types within a given sample.

\subsubsection{Pathway Scoring}

The most significantly different genes (unadjusted $\mathrm{p}$ value $<0.05$ ) were chosen for canonical pathway analysis (Ingenuity Pathway Analysis, Qiagen, Redwood City, Ca. USA).

\subsubsection{Statistics: Diagnostic Modeling}

A machine learning algorithm developed by the NanoString DX group based on Elastic Net was used to define models for predicting adenocarcinoma or benign nodule. Cross-validation was used to select the strength of each penalty, and 1000 loops of cross validation were run for each round of training to estimate the AUC. Multiple rounds of training were performed to optimize signature performance with the input as the $\log 2$ transformed normalized expression data, curated to exclude noise. The output of the optimization was the linear predictor score $=$ $\sigma \chi \beta i ́$ where $\chi=$ gene expression level and $\beta=$ weight.

\subsubsection{Comparison with Soluble Mesothelin Related Peptide (SMRP)}

SMRP is the most commonly used available serum biomarker for MPM diagnosis. Available sera from the patients had serum mesothelin measurement (R\&D, Minneapolis, MN) determined. Our laboratory has measured both serum mesothelin and SMRP measurements in the same patients and converted the $\mathrm{ng} / \mathrm{ml}$ levels seen in the 
mesothelin ELISA to nM levels of SMRP.

\section{Results}

\subsection{Patient Demographics}

Buffy coat from 40 MPMs and 44 AEx originally had quality control and normalization to housekeeping genes. Of these, 5 MPMs and 8 AEx were found to be of low quality. Demographics are seen in Table 1.

Table 1. Cohort Demographics.

\begin{tabular}{lll}
\hline & AE & MPM \\
\hline Age & $66+1$ & $65 \pm 2$ \\
Gender & $36 \mathrm{M}$ & $20 \mathrm{M} ; 15 \mathrm{~F}$ \\
Pack-Years & $23+3$ & $10 \pm 2$ \\
Asbestos Exposure (years) & $40+1$ & 24 yes; 11 no \\
Stage & & Stage I,11; Stage II, 11; Stage III,13 \\
Histology & Epithelial, 29; Biphasic, 4; \\
Surgery & Sarcomatoid, 2 \\
Chemotherapy & None, 1; PD, 32; EPP, 2 \\
Abbreviations: PD, pleurectomy decortication; EPP, extrapleural \\
pneumonectomy
\end{tabular}

As seen in Table 1, all steamfitters had asbestos exposure (range 22-48 years), and 69\% of the MPMs had a history of exposure. The majority of females did not recount a history of fiber exposure. MPMs were equally divided by stage, and the majority had adjuvant chemotherapy.

\subsection{Differential Gene Expression}

Table 2. Differential Gene Expression: AEx Expression/MPM Expression.

\begin{tabular}{lllll}
\hline Gene & $\begin{array}{l}\text { Linear fold } \\
\text { change }\end{array}$ & $\begin{array}{l}\text { 95\% Confidence } \\
\text { Intervals }\end{array}$ & $\begin{array}{l}\text { Unadjusted } \\
\text { p-value }\end{array}$ & FDR \\
\hline IFI27 & 0.176 & $0.0923+0.334$ & 0.00000132 & 0.00616 \\
IL1R2 & 0.367 & $0.245+0.548$ & 0.00000638 & 0.0112 \\
FUT7 & 0.461 & $0.336+0.631$ & 0.000008 & 0.0112 \\
MEF2C & 1.38 & $1.21+1.57$ & 0.0000114 & 0.0112 \\
TGFB1 & 0.591 & $0.475+0.735$ & 0.000012 & 0.0112 \\
CD63 & 0.558 & $0.433+0.719$ & 0.0000266 & 0.0178 \\
CD59 & 0.639 & $0.526+0.777$ & 0.0000271 & 0.0178 \\
ISG15 & 0.405 & $0.272+0.603$ & 0.0000324 & 0.0178 \\
CD44 & 0.558 & $0.431+0.722$ & 0.0000344 & 0.0178 \\
TAPBP & 0.531 & $0.398+0.709$ & 0.0000562 & 0.0245 \\
HLA-DRB3 & 0.507 & $0.372+0.692$ & 0.0000578 & 0.0245 \\
SH2D1B & 1.52 & $1.24+1.85$ & 0.000104 & 0.0403 \\
CD1D & 1.32 & $1.16+1.52$ & 0.000127 & 0.0421 \\
HLA-DPA1 & 1.68 & $1.31+2.15$ & 0.000127 & 0.0421 \\
CD244 & 1.84 & $1.37+2.48$ & 0.000156 & 0.0486 \\
\hline
\end{tabular}

There were 155 differentially expressed genes between the AEx BC and MPM BC with unadjusted p-values $<0.05$. As seen in Table 1, 15 of these met an FDR of 0.05 or less. This is also depicted in the volcano plot in Figure 1.

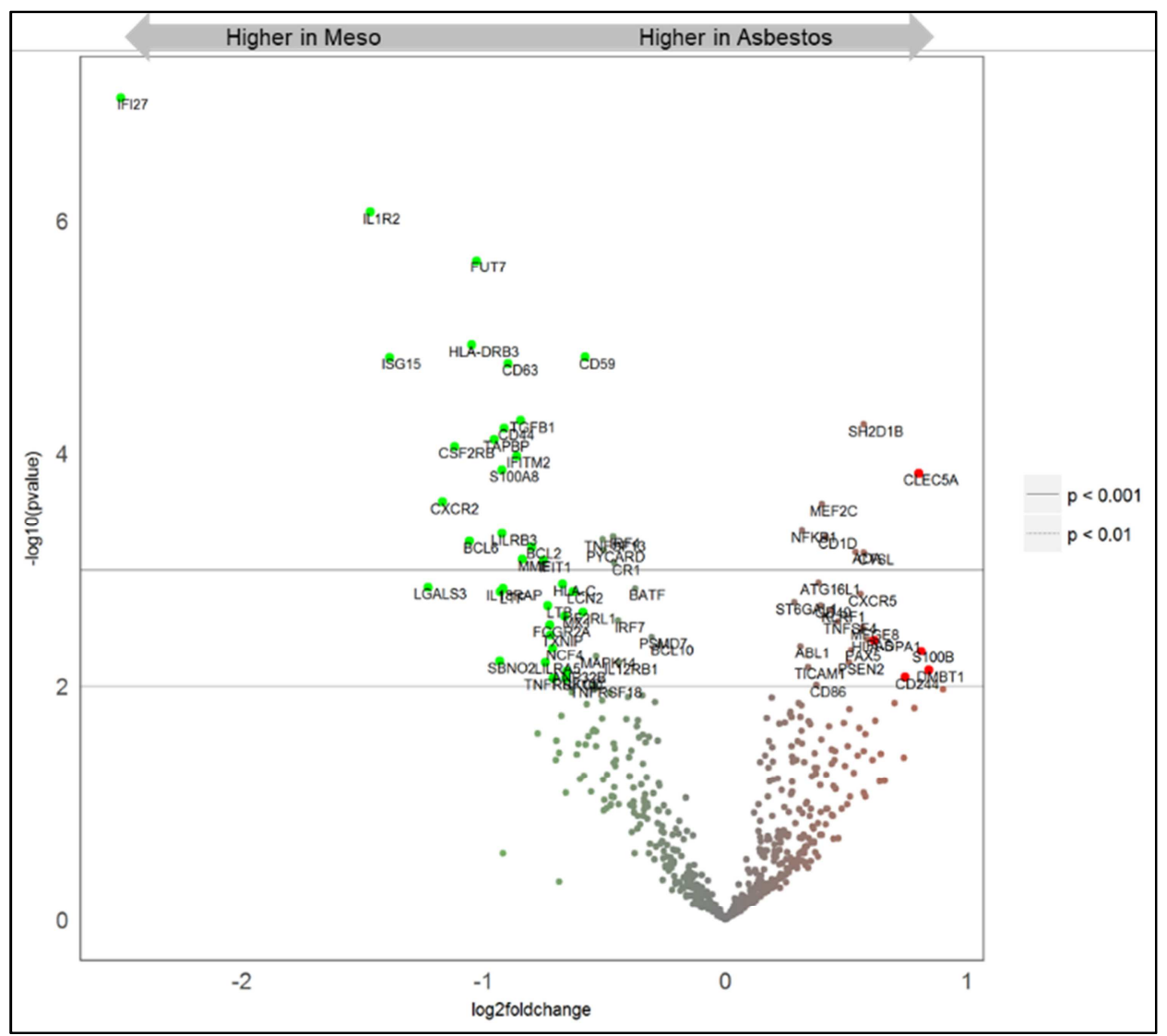

Figure 1. Volcano plot depicting $\log 10$ (p-value) and $\log 2$ fold change. Colored data points and horizontal lines indicate various statistical thresholds. Genes with p-value $<0.01$ are named. Genes with fold change $>1.5$ have enlarged color dots. 


\subsection{Cell Type Immunophenotyping}

Figure 2 illustrates the immune phenotypes in the BCs comparing AEx vs MPMs. Overall, there is a generalized immunosuppressive environment present in the MPMs, with a relative decrease in cytotoxic cells and CD4+ helper T cells, along with decreases in dendritic cells, and mature natural killer cells. Moreover, relative to the AEx, the MPM BC have an increase in exhausted CD8 $+\mathrm{T}$ cells, as well as an increase in neutrophils.

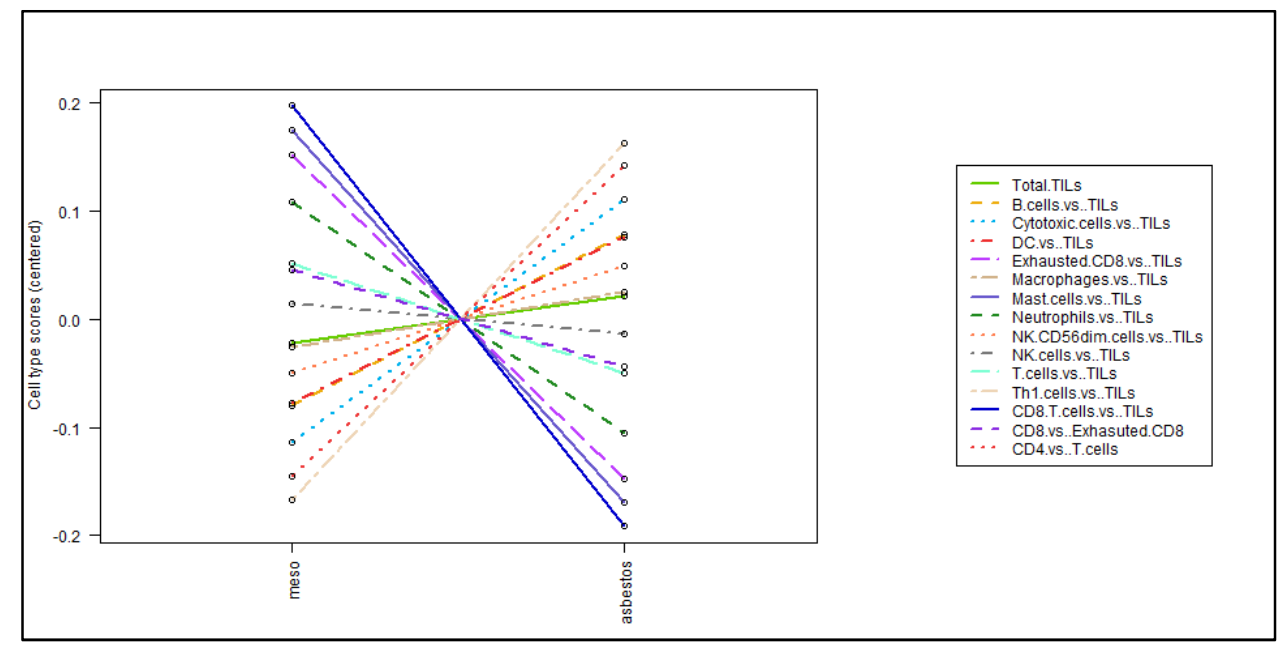

Figure 2. Differences in BC cell type composition between MPM and AEx BC. See text for details.

These data are supported by individual gene comparisons seen in Figure 3. PD-L2 gene expression is seen in the BC of MPMs as well as elevated levels of T-Regs as inferred from FOXP3 expression. TGFB expression, which maintains immune tolerance through lymphocyte proliferation, differentiation, and survival was also significantly elevated in the MPM BC.

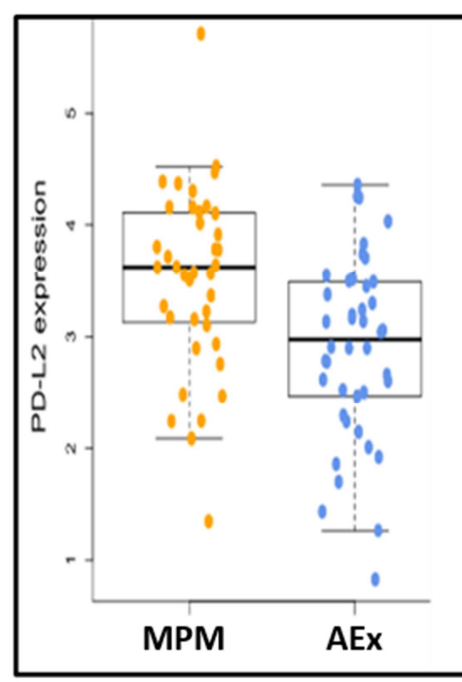

(a)

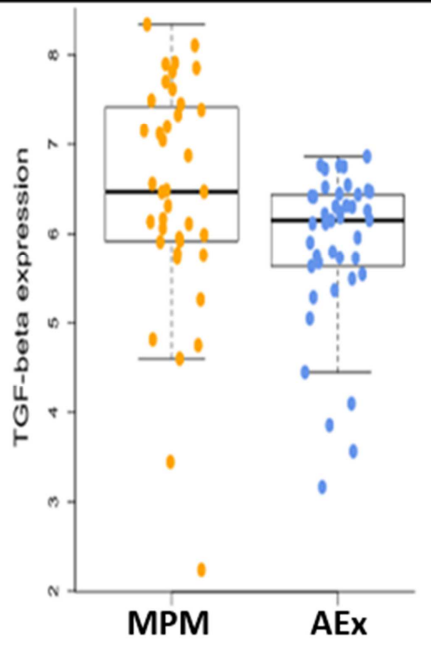

(b)

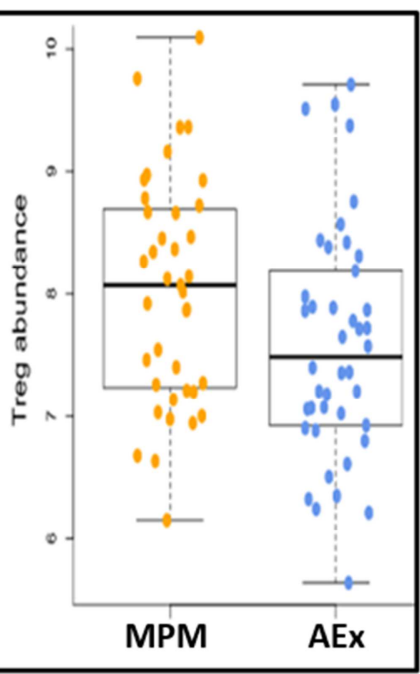

(c)

Figure 3. Further examples of immune suppressive BC environment with elevations of PD-L2, TGF $\beta$, and Tregs.

\subsection{Pathway Analyses}

Figure 4 details the canonical pathways which are associated with gene expression buffy coat differences between MPM and AEx. These pathways reinforce the development of an immunosuppressive environment which evolves in the circulating microenvironment in MPM patients. Specifically, improper immune responses against self-antigens with $\mathrm{B}$ cell differentiation and loss of tolerance is combined with defects in the differentiation of T-cells. Moreover, exhaustion of CD8+ T cells results in production of IL-2, loss of proliferative and cytolytic activity and deficiencies in the TNF $\alpha$, IFN $\gamma$ chemokines, and degranulation, eventually leading to death due to overstimulation. Furthermore, lack of dendritic cell maturation results in failure of co-stimulatory molecule upregulation and MHC Class I and II molecules with resulting 
failures in antigen presentation. The loss of dendritic maturation also downregulates cytokine production and ability to cross present exogenous antigen to cytotoxic T lymphocytes. Finally, aberrant STAT3 signaling promotes initiation and progression of cancers by either inhibiting apoptosis or inducing cell proliferation, angiogenesis, invasion, and metastasis.

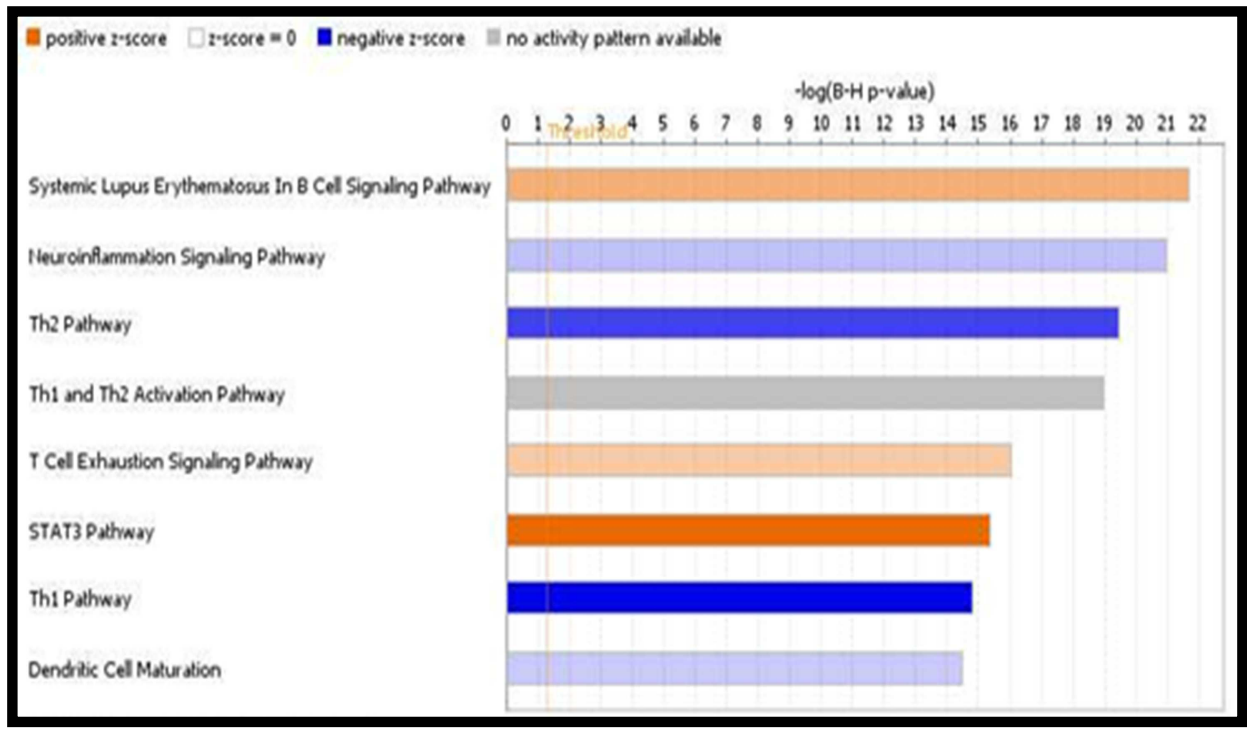

Figure 4. Canonical pathway differences between MPM and AEx BCs.

\subsection{Diagnostic Signature Training}

A finalized gene signature revealed an AUC of 0.99 in the training set of 36 AEx and 35 MPM, and was composed of 37 genes. The gene set, along with their signature weights are seen in Figure 5. Heat map representation of the signature is depicted in Figure 6.

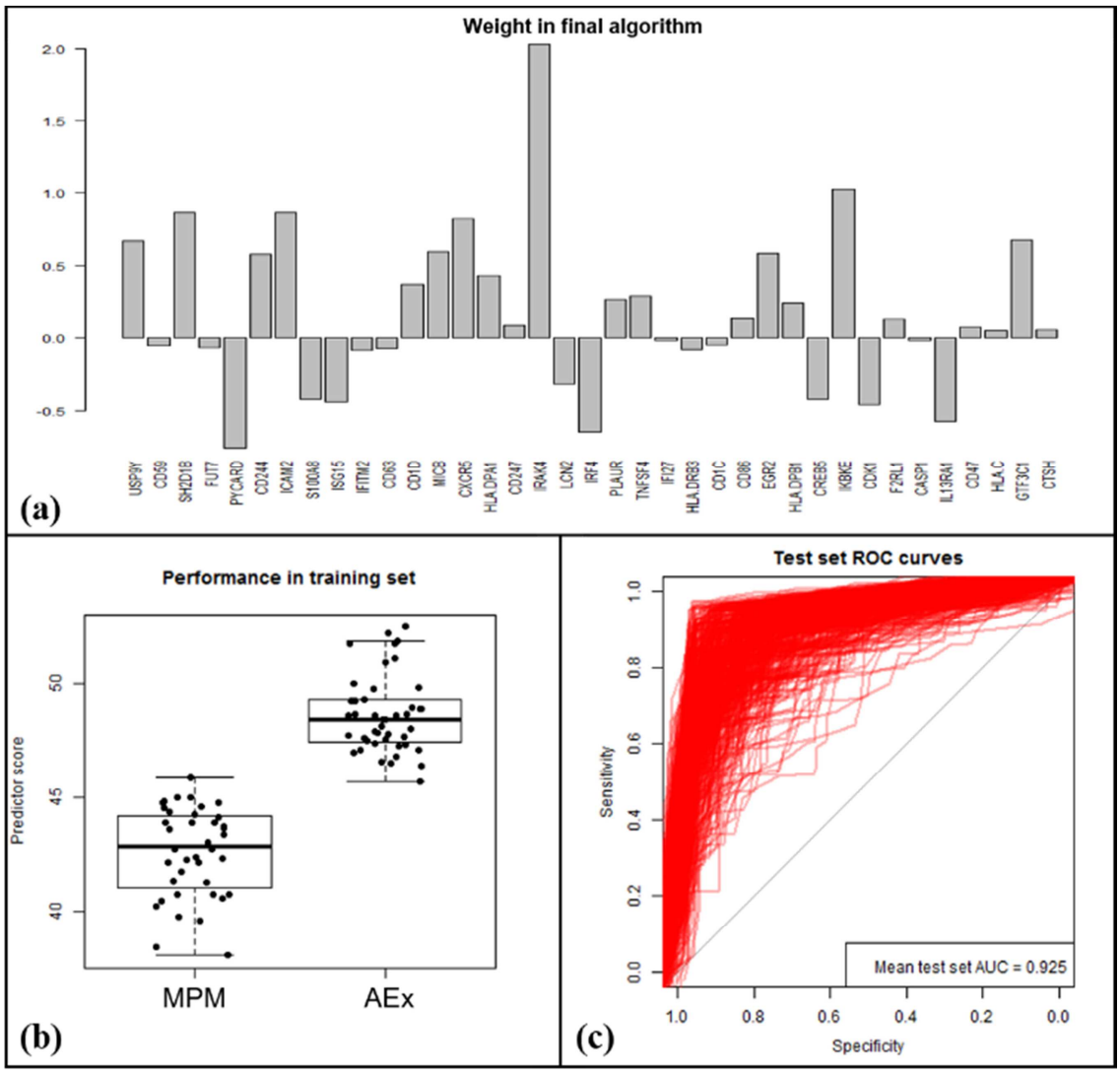

Figure 5. Diagnostic signature development: (a) 37 gene signature with their weights used for signature as described in Section 2.2.5. (b) Interactive bar plots detailing the difference in MPM and AEx by signature scores. (c) Validation loops revealed a mean cross validation of 0.925, CI $=0.81-0.98$. 


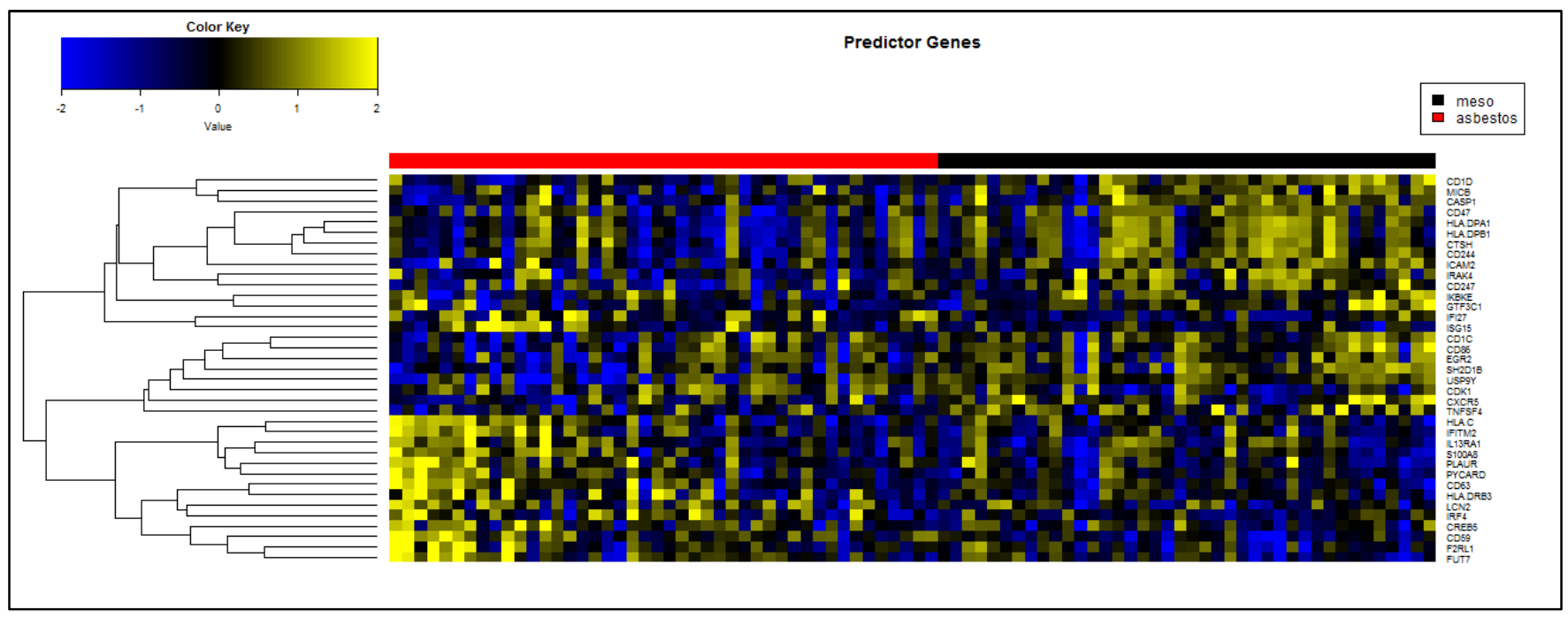

Figure 6. Heat map for discrimination of MPM and AEx BCs using the 37 gene signature.

\subsection{Comparison of BC Immunotranscriptomic Signature with SMRP}

Serum was available on 70/71 individuals from whom the BC immunosignature was constructed. The ROC for estimated SMRP levels comparing the 35 MPM sera to 35 AEx is seen in Figure 7, with an AUC of 0.74.

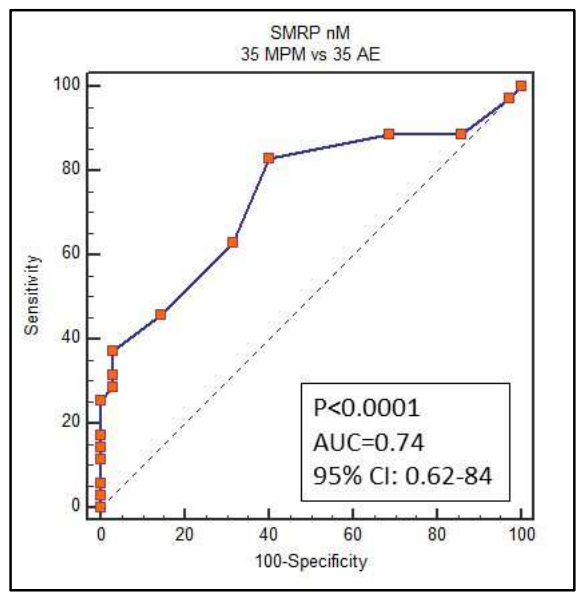

Figure 7. SMRP ROC comparing AEx to MPM.

\section{Discussion}

To the best of our knowledge, our experimental approach differs from the bulk of previous diagnostic mesothelioma biomarker attempts in three significant aspects: (1) The use of a blood-based biosignature. We believe such circulating microenvironment can provide a comprehensive snapshot of mesothelioma immuno-oncology. Buffy coat is processed as the complete fraction of "white cells" from whole blood after centrifugation and represents a heterogeneous mix of circulating leukocytes and platelets. PBMCs are a more homogeneous cellular concentrate of circulating lymphocytes and macrophage, devoid of granulocytes and platelets, and therefore may not reflect the true landscape of immune, inflammatory, and thrombotic processes involved in tumorigenesis. (2) The NanoString platform was our profiling technology of choice. NanoString offers digital PCR with profiling of over 730 known immune related genes through the PanCancer Immune Assay, including those for cytokines and chemokines. Data for analysis are ready in as little as 48 hours from hybridization, and 12 samples can be processed simultaneously. Unlike traditional RNAseq, Nanostring does not require high quality RNA samples or amplification. The sacrifice is doing such an assay is that immunophenotyping of immune cells relies on published algorithms instead of direct identification of a specific immune cell type, i.e., Flow Cytometry (FACS). FACS studies are a common investigative method in the field of immuno-oncology and the development of immunotherapeutics. FACS analyses can phenotype cells with up to 27 parameters; however, it does not provide a global view of all possible proteins/genes involved in the circulating microenvironment. For proof of principle, we felt Nanostring immunoprofiling was adequate. (3) Comparing MPM against a high-risk cohort with prolonged exposure to asbestos. Gene expression profiles found in the majority of MPM biomarker literature are regularly generated from comparisons between MPM and healthy pleural tissue, lung cancer, or in several instances, from MPM alone. We believe the use of high-risk patients is a meaningful step towards clinical utility.

Our data is consistent with the known immunosuppressive oncology of mesothelioma [21], which we believe further supports the validity of our novel approach. Relative to asbestos exposure, we observe the BC transcriptome in MPM is characterized by a tumorigenic and immunosuppressive state that is evident not only in the analysis of differentially expressed genes but through analysis of altered canonical pathways. Differentially expressed genes relatively upregulated in mesothelioma included genes involved in signal transduction (CD63 [22]), cell adhesion and invasion (CD44 [23], FUT7 [24]), protein metabolism (ISG15 [25] immune suppression and escape (TGF $\beta$ [26], CD59 [27]), and inflammation (IL1R2 [28], IFI27 [29]). In contrast, the BC 
transcriptome in asbestos exposure is characterized by a relatively tumor suppressive and immunosupportive state. Differentially expressed genes relatively upregulated in AEx included genes involved in humoral response (MEF2C [30]) and cytotoxic activity (SH2D1B/EAT-2 [31], CD1D [32], CD244 [33]). Immunophenotyping of all differentially expressed genes in MPM BC demonstrated a relative decrease in cytotoxic cells and CD4+ helper $\mathrm{T}$ cells, along with decreases in dendritic cells, and mature natural killer cells. Moreover, relative to the AEx, the MPM BC had an increase in exhausted CD8 $+\mathrm{T}$ cells, as well as an increase in neutrophils. Lastly, gene set analysis of our 37 gene signature model found significant differences in pathways related $\mathrm{T}$ cell activation, differentiation, proliferation, and exhaustion, as well as antigen presenting cell maturation and presentation.

Several of the genes highly expressed in MPM BC relative to AEx (Table 2) encode proteins implicated in cancer development and may be of clinical interest in future studies, particularly as therapeutic targets. CD63 is a membrane surface marker classically found on exosomes [34]. The oncogenic role of exosomes includes their ability to increase the number of immunosuppressive cells, such as regulatory $\mathrm{T}$ cells or inhibitory myeloid cells, or decrease the number of cytotoxic $\mathrm{T}$ cells, natural killer cells and antigen presenting cells [34-36]. Exosomes derived from MPM tumor cells contain oncogenic cargo proteins associated with angiogenesis, cell migration, metastasis, as well as the ability to regulate the tumor microenvironment [37]. The expression of CD44, a multifunctional surface receptor, is associated with the presence of neoplastic mesothelioma cells [38] and has demonstrated the ability to differentiate MPM from other malignancies $[39,40]$. The interaction of CD44 with its ligand, hyaluronan has demonstrated an important role in modulating cell proliferation and invasiveness in MPM [23, 41]. In addition, CD44 is a putative marker of cancer stem cells and may be able to identify chemoresistant subpopulations. ISG15 is a ubiquitin-like protein understood to interfere with protein degradation [25]. Although its precise role in tumorigenesis is still unclear, dysregulation of ISG15 has been implicated in numerous oncogenic and immunomodulatory processes [42]. Various chemotherapeutic agents have been shown to increase ISG15, and ISG15 expression has been shown to be associated with tumor response to chemotherapy and radiotherapy [43, $44]$, as well as the survival of patients with various cancers [45, 46]. Upregulation of CD59, a cell membrane glycoprotein, was reported in a prior mesothelioma transcriptome study [47]. CD59 is potent inhibitor of the complement attack complex action and protects malignant cells from C-mediated lysis [27]. CD59 exerts a downmodulatory effect on T lymphocytes and influences cytokine production in the immune microenvironment [48-50]. Increased expression of CD59 is associated with worse clinical outcomes in several cancers [51, 52]. Blockade of CD59 has been shown to enhance $\mathrm{T}$ cell response [48] and therapeutic effect $[53,54]$. Overexpression of IL1R2, a decoy receptor of exogenous IL-1, has been observed in numerous cancers [55-57]. The gene signature of activated tumor Tregs, which included IL1R2, correlated with poor prognosis in lung adenocarcinoma [58]. Breast cancer patients with high IL1R2 expression in tissue have a poorer overall survival and relapse-free survival. Blockade IL1R2 blockade suppressed breast tumorigenesis and progression [59]. TAPBP (or Tapasin) is a transmembrane glycoprotein that mediates optimal peptide loading on MHC class 1 molecules and thus promotes tumor antigen presentation and recognition by $\mathrm{T}$ cells [60]. Defects in TAPBP expression are associated with tumor progression. In contrast, upregulation of TAPBP has been associated increased tumor immunogenicity following pharmacological intervention and has been implicated in drug response [61, 62]. Overexpression of TAPBP in our MPM cohort may have been influenced by history of chemotherapy.

We have shown that it is feasible to use a BC transcriptomic signature to differentiate asbestos exposed individuals from those with MPM, and that this 37 gene signature is at least comparable to the most commonly used blood-based diagnostic biomarker for MPM, i.e., SMRP. Moreover, the differences between the BCs describe an immunosuppressive conversion in the blood microenvironment as well as one that promotes tumor growth and invasion. There are, however, obvious limitations which mandate further work regarding our findings. Internal validations are not nearly as definitive as validation of molecular signatures with other cohorts. Buffy coat is not the most common analyte used for these analyses, which makes cross referencing our results with the literature challenging. The description and cataloging of all the genes which of significance in these analyses is beyond the limits of this presentation; however, beyond validating molecular signature, further exploration of how these genes influence recurrence or differ between benign and malignant lesions needs to be investigated in mechanistic experiments. As stated earlier, despite the multitude of genes surveyed in these investigations we cannot definitively characterize the individual immune phenotypes as specifically as fluorescence sorting could. Therefore, any interpretation of which pathways are reflected by the interaction of these genes depends on the "state of the art" in the literature at the time of the analysis. Finally, the gene expression profile of patient's pretreatment (surgical or chemo) may differ posttreatment [63, 64]. Given that our models were trained and tested on a mixture of both pre- and post-treatment, applicability to largely pretreatment populations in early screening and diagnostic contexts may vary.

\section{Conclusions}

We conclude that the buffy coat represents a novel circulating microenvironment to explore for screening and early diagnosis of mesothelioma. Buffy Coat immunotranscriptomics accurately differentiated between MPM and high-risk asbestos exposure with a 37 gene signature. The diagnostic accuracy of our buffy coat immunotranscriptomic signature is at least comparable to the most commonly used blood-based diagnostic biomarker for MPM, serum mesothelin-related peptide. In addition, 
immunophenotyping and pathway analysis of 155 differentially expressed genes characterized MPM buffy coat as a relatively tumorigenic and immunosuppressive state. Several of the most differentially expressed genes encode proteins implicated in cancer development (e.g., CD63, CD44, ISG15, CD59, IL1R2, and TAPBP) and may hold clinical value as therapeutic targets. Larger, externally validated studies are needed to refine the signature for clinical relevance and develop a more robust diagnostic panel.

\section{Acknowledgements}

We acknowledge the support of Wenje $\mathrm{Xu} \mathrm{PhD}$, Nanostring who provided excellent biostatistical analyses on a contractual basis with NYU Langone Health.

\section{References}

[1] Brusselmans, L., et al., Breath analysis as a diagnostic and screening tool for malignant pleural mesothelioma: a systematic review. Translational Lung Cancer Research, 2018. 7 (5): p. 520-536.

[2] Carbone, M., et al., Consensus Report of the 2015 Weinman International Conference on Mesothelioma. Journal of thoracic oncology: official publication of the International Association for the Study of Lung Cancer, 2016. 11 (8): p. 1246-1262.

[3] Tsim, S., et al., The diagnostic performance of routinely acquired and reported computed tomography imaging in patients presenting with suspected pleural malignancy. Lung Cancer, 2017. 103: p. 38-43.

[4] Tsao, M. S., et al., Pathologic Considerations and Standardization in Mesothelioma Clinical Trials. J Thorac Oncol, 2019. 14 (10): p. 1704-1717.

[5] Tsao, A. S., et al., Current and Future Management of Malignant Mesothelioma: A Consensus Report from the National Cancer Institute Thoracic Malignancy Steering Committee, International Association for the Study of Lung Cancer, and Mesothelioma Applied Research Foundation. J Thorac Oncol, 2018. 13 (11): p. 1655-1667.

[6] Schreiber, R. D., L. J. Old, and M. J. Smyth, Cancer Immunoediting: Integrating Immunity's Roles in Cancer Suppression and Promotion. Science, 2011. 331 (6024): p. 1565-1570.

[7] Chaussabel, D., Assessment of immune status using blood transcriptomics and potential implications for global health. Seminars in Immunology, 2015. 27 (1): p. 58-66.

[8] Holsbø, E., et al., Predicting breast cancer metastasis from whole-blood transcriptomic measurements. BMC Research Notes, 2020. 13: p. 1-5.

[9] Zander, T., et al., Blood-Based Gene Expression Signatures in Non-Small Cell Lung Cancer. Clinical Cancer Research, 2011. 17 (10): p. 3360-3367.

[10] Aarøe, J., et al., Gene expression profiling of peripheral blood cells for early detection of breast cancer. Breast Cancer Research, 2010. 12 (1): p. R7.
[11] Showe, M. K., et al., Gene expression profiles in peripheral blood mononuclear cells can distinguish patients with nonsmall cell lung cancer from patients with nonmalignant lung disease. Cancer research, 2009. 69 (24): p. 9202-9210.

[12] Baine, M. J., et al., Transcriptional profiling of peripheral blood mononuclear cells in pancreatic cancer patients identifies novel genes with potential diagnostic utility. PloS one, 2011.6 (2): p. e17014.

[13] Ciarloni, L., et al., Development and clinical validation of a blood test based on 29-gene expression for early detection of colorectal cancer. Clinical Cancer Research, 2016. 22 (18): p. 4604-4611.

[14] Eruslanov, E. B., Phenotype and function of tumor-associated neutrophils and their subsets in early-stage human lung cancer. Cancer Immunol Immunother, 2017. 66 (8): p. 997-1006.

[15] Faget, J., et al., Neutrophils and Snail Orchestrate the Establishment of a Pro-tumor Microenvironment in Lung Cancer. Cell Rep, 2017.21 (11): p. 3190-3204.

[16] Mansour, A., et al., P2Y12 Inhibition beyond Thrombosis: Effects on Inflammation. Int J Mol Sci, 2020. 21 (4).

[17] Maurer, S., et al., Platelet-mediated shedding of NKG2D ligands impairs NK cell immune-surveillance of tumor cells. Oncoimmunology, 2018.7 (2): p. e1364827.

[18] Cesano, A., nCounter (R) PanCancer Immune Profiling Panel (NanoString Technologies, Inc., Seattle, WA). J Immunother Cancer, 2015. 3: p. 42.

[19] Jerby-Arnon, L., et al., A Cancer Cell Program Promotes T Cell Exclusion and Resistance to Checkpoint Blockade. Cell, 2018. 175 (4): p. 984-997.e24.

[20] Vandesompele, J., et al., Accurate normalization of real-time quantitative RT-PCR data by geometric averaging of multiple internal control genes. Genome Biology, 2002. 3 (7): p. research 0034.1.

[21] Yap, T. A., et al., Novel insights into mesothelioma biology and implications for therapy. Nature Reviews Cancer, 2017. 17 (8): p. 475.

[22] Ahmadzada, T., et al., Extracellular vesicles as biomarkers in malignant pleural mesothelioma: A review. Critical reviews in oncology/hematology, 2020: p. 102949.

[23] Marhaba, R. and M. Zöller, CD44 in Cancer Progression: Adhesion, Migration and Growth Regulation. Journal of Molecular Histology, 2004. 35 (3): p. 211-231.

[24] Liang, J.-X., W. Gao, and L. Cai, Fucosyltransferase VII promotes proliferation via the EGFR/AKT/mTOR pathway in A549 cells. OncoTargets and therapy, 2017. 10: p. 3971-3978.

[25] Desai, S. D., et al., Elevated expression of ISG15 in tumor cells interferes with the ubiquitin/26S proteasome pathway. Cancer research, 2006. 66 (2): p. 921-928.

[26] Digifico, E., et al., Microenvironment and immunology of the human pleural malignant mesothelioma, in Mesothelioma. 2019, Springer. p. 69-84.

[27] Zhang, R., et al., CD59: a promising target for tumor immunotherapy. Future Oncology, 2018. 14 (8): p. 781-791. 
[28] Kadariya, Y., et al., Inflammation-related IL1 $\beta / I L 1 R$ signaling promotes the development of asbestos-induced malignant mesothelioma. Cancer Prevention Research, 2016. 9 (5): p. 406-414.

[29] Cheriyath, V., D. W. Leaman, and E. C. Borden, Emerging roles of FAM14 family members (G1P3/ISG 6-16 and ISG12/IFI27) in innate immunity and cancer. Journal of Interferon \& Cytokine Research, 2011.31 (1): p. 173-181.

[30] Wilker, P. R., et al., Transcription factor Mef2c is required for $\mathrm{B}$ cell proliferation and survival after antigen receptor stimulation. Nature immunology, 2008.9 (6): p. 603.

[31] Roncagalli, R., et al., Negative regulation of natural killer cell function by EAT-2, a SAP-related adaptor. Nature Immunology, 2005. 6 (10): p. 1002-1010.

[32] Tagawa, T., et al., Antitumor Impact of Interferon- $\gamma$ Producing CD1d-restricted NKT Cells in Murine Malignant Mesothelioma. Journal of Immunotherapy, 2013. 36 (8): p. 391-399.

[33] Agresta, L., K. H. N. Hoebe, and E. M. Janssen, The Emerging Role of CD244 Signaling in Immune Cells of the Tumor Microenvironment. Frontiers in Immunology, 2018. 9 (2809).

[34] Bobrie, A., et al., Exosome secretion: molecular mechanisms and roles in immune responses. Traffic, 2011. 12 (12): p. $1659-1668$.

[35] Vulpis, E., et al., Cancer exosomes as conveyors of stress-induced molecules: new players in the modulation of NK cell response. International journal of molecular sciences, 2019. 20 (3): p. 611.

[36] Xu, R., et al., Extracellular vesicles in cancer-implications for future improvements in cancer care. Nature reviews Clinical oncology, 2018. 15 (10): p. 617-638.

[37] Creaney, J., et al., A Proteomic Analysis of the Malignant Mesothelioma Secretome Using iTRAQ. Cancer Genomics Proteomics, 2017. 14 (2): p. 103-117.

[38] Penno, M., et al., High CD44 expression on human mesotheliomas mediates association with hyaluronan. The cancer journal from Scientific American, 1995. 1 (3): p. 196-203.

[39] Porcel, J. M., et al., The use of pleural fluid sCD44v6/std ratio for distinguishing mesothelioma from other pleural malignancies. Journal of Thoracic Oncology, 2011. 6 (1): p. 190-194.

[40] Afify, A. M., R. Stern, and C. W. Michael, Differentiation of mesothelioma from adenocarcinoma in serous effusions: the role of hyaluronic acid and CD44 localization. Diagnostic cytopathology, 2005. 32 (3): p. 145-150.

[41] Jothy, S., CD44 and its partners in metastasis. Clinical \& experimental metastasis, 2003. 20 (3): p. 195-201.

[42] Han, H. G., H. W. Moon, and Y. J. Jeon, ISG15 in cancer: Beyond ubiquitin-like protein. Cancer Letters, 2018. 438: p. $52-62$.

[43] Weichselbaum, R. R., et al., An interferon-related gene signature for DNA damage resistance is a predictive marker for chemotherapy and radiation for breast cancer. Proc Natl Acad Sci U S A, 2008. 105 (47): p. 18490-5.
[44] Liu, M., et al., Camptothecin induces the ubiquitin-like protein, ISG15, and enhances ISG15 conjugation in response to interferon. Journal of interferon \& cytokine research, 2004. 24 (11): p. 647-654.

[45] Bektas, N., et al., The ubiquitin-like molecule interferon-stimulated gene 15 (ISG15) is a potential prognostic marker in human breast cancer. Breast Cancer Research, 2008. 10 (4): p. 1-12.

[46] Darb-Esfahani, S., et al., Interferon-stimulated gene, $15 \mathrm{kDa}$ (ISG15) in ovarian high-grade serous carcinoma: prognostic impact and link to NF- $\mathrm{kB}$ pathway. International Journal of Gynecological Pathology, 2014. 33 (1): p. 16-22.

[47] Rihn, B., et al., Differential gene expression in mesothelioma. FEBS letters, 2000. 480 (2-3): p. 95-100.

[48] Sivasankar, B., et al., CD59 blockade enhances antigen-specific $\mathrm{CD} 4+\mathrm{T}$ cell responses in humans: a new target for cancer immunotherapy? The Journal of Immunology, 2009. 182 (9): p. 5203-5207.

[49] Xie, X.-H., et al., Post-transcriptional CD59 gene silencing by siRNAs induces enhanced human $\mathrm{T}$ lymphocyte response to tumor cell lysate-loaded DCs. Cellular Immunology, 2012. 274 (1): p. 1-11.

[50] Lipp, A. M., et al., Lck mediates signal transmission from CD59 to the TCR/CD3 pathway in Jurkat T cells. PLoS One, 2014. 9 (1): p. e85934.

[51] Song, G., et al., Increased CD59 protein expression is associated with the outcome of patients with diffuse large B-cell lymphoma treated with R-CHOP. Medical Oncology, 2014. 31 (7): p. 56.

[52] Xu, C., et al., Increased CD59 protein expression predicts a PSA relapse in patients after radical prostatectomy. The Prostate, 2005. 62 (3): p. 224-232.

[53] Zhao, W.-P., et al., Neutralization of complement regulatory proteins CD55 and CD59 augments therapeutic effect of herceptin against lung carcinoma cells. Oncol Rep, 2009. 21 (6): p. 1405-1411.

[54] You, T., et al., Application of a novel inhibitor of human CD59 for the enhancement of complement-dependent cytolysis on cancer cells. Cellular \& Molecular Immunology, 2011. 8 (2): p. 157-163.

[55] Ma, Y., et al., Proteomics analysis of Hodgkin lymphoma: identification of new players involved in the cross-talk between HRS cells and infiltrating lymphocytes. Blood, 2008. 111 (4): p. 2339-2346.

[56] Mar, A.-C., et al., Interleukin-1 Receptor Type 2 Acts with c-Fos to Enhance the Expression of Interleukin-6 and Vascular Endothelial Growth Factor A in Colon Cancer Cells and Induce Angiogenesis*. Journal of Biological Chemistry, 2015. 290 (36): p. 22212-22224.

[57] Rückert, F., et al., Examination of apoptosis signaling in pancreatic cancer by computational signal transduction analysis. PloS one, 2010.5 (8): p. e12243.

[58] Guo, X., et al., Global characterization of $\mathrm{T}$ cells in non-small-cell lung cancer by single-cell sequencing. Nature Medicine, 2018. 24 (7): p. 978-985. 
[59] Zhang, L., et al., IL1R2 Blockade Suppresses Breast Tumorigenesis and Progression by Impairing USP15-Dependent BMI1 Stability. Advanced Science, 2020. 7 (1): p. 1901728.

[60] Leone, P., et al., MHC class I antigen processing and presenting machinery: organization, function, and defects in tumor cells. J Natl Cancer Inst, 2013. 105 (16): p. 1172-87.

[61] Teo, Z. L., et al., Combined CDK4/6 and PI3K $\alpha$ Inhibition Is Synergistic and Immunogenic in Triple-Negative Breast Cancer. Cancer Res, 2017. 77 (22): p. 6340-6352.
[62] Goel, S., et al., CDK4/6 inhibition triggers anti-tumour immunity. Nature, 2017. 548 (7668): p. 471-475.

[63] Schrump, D. S., et al., Phase I Study of Decitabine-Mediated Gene Expression in Patients with Cancers Involving the Lungs, Esophagus, or Pleura. Clinical Cancer Research, 2006. 12 (19): p. 5777-5785.

[64] Sidi, R., et al., Induction of senescence markers after neo-adjuvant chemotherapy of malignant pleural mesothelioma and association with clinical outcome: An exploratory analysis. European Journal of Cancer, 2011. 47 (2): p. 326-332. 